\title{
Ethnologies
}

\section{Bijoux à secrets. Par Patrizia Ciambelli (Paris : Éditions de la Maison des sciences de l'homme, Collection Ethnologie de la France, 2002. 144p., ISBN 2-73510945-3)}

\section{Christine Bricault}

Volume 25, numéro 1, 2003

Négocier la transcendance / Negotiating Transcendence

URI : https://id.erudit.org/iderudit/007142ar

DOI : https://doi.org/10.7202/007142ar

Aller au sommaire du numéro

Éditeur(s)

Association Canadienne d'Ethnologie et de Folklore

ISSN

1481-5974 (imprimé)

1708-0401 (numérique)

Découvrir la revue

Citer ce compte rendu

Bricault, C. (2003). Compte rendu de [Bijoux à secrets. Par Patrizia Ciambelli (Paris : Éditions de la Maison des sciences de l'homme, Collection Ethnologie de la France, 2002. 144p., ISBN 2-73510945-3)]. Ethnologies, 25(1), 297-299.

https://doi.org/10.7202/007142ar d'utilisation que vous pouvez consulter en ligne.

https://apropos.erudit.org/fr/usagers/politique-dutilisation/ 
Bijoux à secrets. Par Patrizia Ciambelli (Paris : Éditions de la Maison des sciences de l'homme, Collection Ethnologie de la France, 2002. 144p., ISBN 2-73510945-3)

Bijoux à secrets est un ouvrage qui porte exclusivement sur la place et le rôle des bijoux dans la vie des femmes et des hommes d'aujourd'hui. Il s'inscrit dans la foulée des préoccupations actuelles sur le corps et l'apparence. Ethnologue de formation, Patrizia Ciambelli est conservatrice au Musée national des arts et traditions populaires de Rome et chercheure associée au Centre d'Anthropologie de Toulouse. L'ouvrage paru dans la collection Ethnologie de la France fait partie de l'une des trois collections de la Mission du Patrimoine ethnologique et s'adresse tant à un large public qu'aux ethnologues, anthropologues, sociologues ou historiens de formation.

Avant tout, il est important de noter le rapport de proximité qui existe entre l'auteure et son objet d'étude. Bijoutier de profession, le père de Patrizia Ciambelli tenait une bijouterie dans le quartier de Santa Lucia à Naples. Toute jeune fascinée par le travail de son père, l'auteure a entrepris des années plus tard de rencontrer «des femmes qui ont accepté d'ouvrir pour [elle] leurs coffrets et de laisser parler leurs bijoux » (vii). En effet, l'étude s'appuie sur des sources de première main recueillies au moyen de l'enquête orale. L'auteure a réalisé des enquêtes en France, en Italie et en Espagne auprès de citoyens de tous milieux et de toutes classes sociales, tout d'abord entre 1993 et 1995 et, par la suite, entre 1996 et 2000. Au total, 304 questionnaires ont été remplis. Bref, il s'agit d'un travail de terrain de grande échelle.

L'étude de Patrizia Ciambelli se base sur la prémisse selon laquelle les bijoux participent en eux-mêmes à la construction identitaire d'un individu. La démonstration de l'auteure suit une progression logique qui témoigne des différents statuts du bijou : du caractère enfantin du bijou jusqu'à l'épineuse question des bijoux de famille. Le volume est divisé en six chapitres de proportion inégale dans lesquels on peut entrer véritablement dans le monde des joyaux.

Le premier chapitre de l'ouvrage, intitulé « Bijoux indiscrets », agit en guise d'introduction. L'auteure expose ses intentions en insistant sur le rôle qu'il importe d'accorder à l'homme, très souvent exclu d'un tel sujet. Elle se propose de chercher, en considérant les bijoux comme témoins de vie, à connaître les règles qui présupposent l'achat, le port et le don de bijoux. 
La section suivante nous plonge véritablement dans le monde du bijou typé : le bijou féminin. L'auteure traite de la première parure de l'enfant : sa médaille de baptême. Elle nous porte à voir le comportement des jeunes filles qui se fabriquent des bijoux. Qui plus est, l'ethnologue laisse parler ses informateurs et cite un récit de nettoyage de bijou fascinant. On y apprend aussi que la première acquisition du bijou se fait par le regard. Les fillettes s'initient donc aux diverses règles implicites et explicites du port des bijoux en observant les membres féminins de la cellule familiale. Finalement, Ciambelli propose la théorie de l'accès graduel selon laquelle l'ordre dans lequel la femme accède au bijou respecte une certaine progression : on passe du métal à la pierre et de l'opacité à la brillance.

«La Boucle et la marque », troisième chapitre du livre, propose de résumer l'histoire de la boucle d'oreille. On y aborde plusieurs sujets, dont les anciennes techniques de perçage, le perçage en tant que rite d'initiation et le perçage et l'amitié. Toutefois, ce qui nous a semblé le plus intéressant de cette section reste la réflexion que propose l'auteure sur la douleur lors du perçage. Cette dernière explique que, jusqu'à un certain point, la souffrance devient un élément de motivation, une expérience du mal. Finalement, Patrizia Ciambelli expose certaines croyances populaires selon lesquelles percer un trou dans l'oreille éclaircirait la vue. La quatrième section, plus difficile à saisir, tend à montrer l'analogie qui peut exister entre la femme et le cheval par l'expression «ferrer l'épouse ». On aborde ici le sujet du mariage et de l'échange d'alliances. Après avoir traité de la fonction et de la signification des bijoux en forme de fer à cheval, l'auteure relate l'importance du forgeron et de la figure de saint Éloi. Ces éléments, quoique semblant se détacher des propos premiers de l'auteure, sont toutefois rapidement rattachés à la recrudescence du phénomène du tatouage dans la société actuelle. Le cinquième volet traite de l'éternelle dualité entre le vrai bijou et le faux. L'auteure résume bien sa réflexion sur le sujet en écrivant, "Les faux ont du sens dans une société qui réclame du vrai » (73). La majeure partie du chapitre est destinée à la perte des bijoux. On y explore également l'aspect de l'abandon volontaire et du vol de bijoux. Habituellement très éprouvantes, ces pertes correspondent souvent au démembrement du patrimoine familial.

Enfin, dans le dernier volet, Patrizia Ciambelli nous fait entrer dans l'univers du bijou destiné à créer et à défaire les liens : le bijou de famille. Rempli d'histoires, de secrets et souvent de frustrations, ce type de 
bijou régularise la vie d'une famille en y marquant les différentes étapes biographiques. Pour l'auteure, la force du bijou réside dans « ces allers et retours sur une frontière toujours mouvante» (115).

La grande originalité de ce livre se situe au niveau de son traitement. La forme de l'ouvrage et le ton qui l'accompagne s'apparentent parfois à la nouvelle. Cet aspect, qui parfois tourne presque au romanesque, surprend et ravit à la fois. Avec une certaine retenue, il est possible d'émettre l'hypothèse que le but de l'auteure était de se faire plaisir et peut-être de rendre hommage à son père. Cette sensibilité, de même que les nombreux exemples tirés des enquêtes orales, provoquent chez le lecteur une réaction d'identification. Quelle petite fille n'a pas accroché des cerises à ses oreilles en guise de premier pendentif et qui n'a jamais été attiré par les trésors que réservait le coffre à bijoux de grand-mère ? En ce sens, le livre joue le rôle d'un véritable recueil de souvenirs. La présentation des données de l'enquête se fait de manière naturelle, les extraits de propos des locuteurs sont abondamment utilisés, si bien que, parfois, on en oublie presque le but premier de l'ouvrage.

Un autre caractère particulièrement pertinent est l'étendue de l'étude qui aborde, sans y aller en profondeur, des thèmes importants liés au sujet comme le piercing, le tatouage, le maquillage et l'utilisation du parfum, autant de formes de modification et d'ornementation du corps. L'ouvrage est abondamment illustré. Ces images, parfois dessins, parfois photographies, objets, tableaux ou affiches publicitaires, appuient remarquablement bien les propos de l'auteure et égayent la lecture.

On regrette cependant la fin quelque peu abrupte de la recherche, dépourvue de conclusion. Il est difficile de synthétiser un ouvrage aussi riche de découvertes et de réflexions. Certes, il est intéressant de noter que Patrizia Ciambelli fait état d'autres études faites sur le sujet. L'importante bibliographie témoigne d'un travail peaufiné et bien mené. L'utilisation des sources littéraires et philosophiques est intelligente et, de ce fait, l'ouvrage mérite d'être lu pour son contenu autant que pour sa forme. Bref, l'ethnologue Patrizia Ciambelli, avec finesse et sensibilité, fait parler le bijou et nous confie ses secrets. 